\title{
Combinatorial conditionality of culture-specific collocations
}

\author{
Elena Arkhipova ${ }^{1, *}$, Marina Vlavatskaya $^{1}$ \\ ${ }^{1}$ Novosibirsk State Technical University, 20, Karl Marx, 630073, Novosibirsk, Russia
}

\begin{abstract}
Combinatorial lexicology as a section of lexicology deals with the theory of syntagms, valency, collocability, the relation of word semantics and collocability, the relation of syntagmatics of a language sign and the language system, functions of collocability, etc. Due to the high importance of linguocultural competence, it comprises such subsection as ethnocultural combinatorial lexicology, which studies ethnocultural collocations - combinatorially conditioned combinations of words reflecting socially significant realities for a certain ethnic group, in other words containing cultural specificity, which impedes their understanding by the representatives of other linguocultures. The article covers the peculiarities of the syntagmatic relations, semantic properties of words to create culture-specific collocations, the role of context in actualization of the meaning of the word and uniqueness of some concepts for a specific language community.
\end{abstract}

\section{Introduction}

Research in the field of combinatorial linguistics is of natural interest to the problem of syntagmatic relations of words as a certain system of signs, characterized by the property of linear length.

The study of the syntagmatic relations of words in both Russian and foreign linguistic traditions begins with syntax due to the availability of observation of the links between syntactic dependencies and the order of elements. Further, the attention turned to the semantic aspect of linguistic units, as a result of which syntax and meaning began to be considered in interrelation.

At present, the basis of combinatorial lexicology consists of the concepts: valency - the potential collocability of language units that takes place at the level of the language, collocability - realized valency, or real collocability in speech, distribution - the totality of all environments of a language unit, and context - one of all environments of a word.

The collocability of a word is inseparable from its semantics; therefore the question of the co-relation of these entities has always remained debatable. In this paper, we recognize collocability as the most important linguistic indicator of meaning, but not the meaning itself [1]. The combinatorial potential of the semantics of the word reveals itself in ethnocultural collocations, in particular, denoting unique concepts of particular community.

\section{Methodology}

In early works on semantics the lexical meaning was presented as an established display of a subject or phenomenon in people's minds. Now the studies are carried out with the understanding of a great complexity of lexical meaning having no clear boundaries. These entities include, along with the main meaning, a complex of associative augments that are realized in speech with a certain set of words in context. The connection of linear related words with the culture of a particular ethnic group gives rise to ethnocultural combinatorial linguistics [2].

The theory of collocations brings the notion of collocational constituent to lexicology, which means the constituent of the word combination, but not always a separate word. For example, the collocation "enter into a pact" consists of four words and only two collocational constituents, because "into a pact" is taken as a whole. To proceed this idea linguists create a notion of a complex collocation [3], which is a combination of a lexeme and an idiom. This term is singled out due to the difference of simple and complex collocations, which is seen in internal complexity of constituents. The Hockett's evolutionary theory of the combinatorial structure of the human language is about combination of not only acoustic building blocks into utterances, but further combinations into meaningful utterances according to some rules [4].

In language and speech, collocability performs various functions, e.g. it demarcates the meanings not only in everyday speech, but also in professional language, where it is difficult enough for a person who is not into some particular area to understand what is being discussed.

Collocability is a way of actualizing semantic features of meaning, which is the correlation of a potential sign with reality, consisting in adapting virtual elements of a language to the requirements of a given speech situation through actualizers [5]. Actualization extends not only to the denotative component of the

\footnotetext{
* Corresponding author: elena1503@inbox.ru
} 
meaning of the word, but also to the potential visualsensual and emotional-evaluative elements of the meaning, e.g. such words as a mountain, an ocean, an iceberg contain the seme of "many", which is actualized, while the semes "large landform", "a body of water", "a piece of freshwater ice" are removed from the meaning of these words. Actualization is a complex phenomenon, usually realized in an expanded statement or text.

Collocability for the speaker and the listener is of particular importance due to the fact that they are related to the text in a completely different way: the speaker goes from meaning to the context, and the listener moves from context to the meaning. The speaker, who provides information, chooses certain language tools. The listener in the process of communication perceives the linguistic form, decodes it, and thus receives information transmitted. In the sentence, "At that very moment, as I stood in the shadows, the green snake bit into my heart", the meaning of the word combination "green snake" means "jealousy". Collocability of words contributes to the demarcation in our consciousness of the heard or read phrases from many other combinations.

Collocability is one of the main methods of creating a comic effect, for example, when collocability, which distinguishes between direct and figurative meanings, is specifically eliminated, and homonyms or paronyms are intentionally used. In these cases, there is a pun, a figure of speech consisting in a humorous use of different meanings of the same word or two similarly sounding words. For example, "A tailor guarantees to give each of his customers a perfect fit", a clash of different meanings of the word occurs, which gives rise to the comic effect of the situation. From this statement, it is easy to understand that the first meaning of the word fit is "perfectly fitting clothes", and the second that is "a nervous spasm".

Therefore, collocability is one of the most important linguistic phenomena, having many functions, contributing both to the demarcation of the meanings of polysemous words, and to the pushing together of the meanings of a polysemous word to achieve the goal of making the statement comic.

Within the framework of the combinatorial lexicology, the recognition of the syntagmatic aspect, responsible for the connection of lexical units in the speech chain, is considered appropriate. This aspect has a rather complicated structure, since includes selective and restrictive components. The first points to the meaningful use of the word in the speech chain, the second is a double restriction of collocability, regulates the further specification within the semantic class of lexemes.

The division of the selective component into subcomponents is due to the different nature of the restrictions on the collocability of a word. The function of the denotative-selective subcomponent is to reflect the usual ideas about objects and phenomena of reality. Limitations in the denotative component of the meaning are well observed in the following syntagmas: to recite poetry, a sun beam, the grass rustling, to cook dinner, etc.
The connotative-selective subcomponent requires, for example, the use of a particular style of speech in a statement. The sentence * The police caught one of the most celebrated thieves last night seems not correct, because the word "celebrated" has positive connotation. It would be more appropriate to make a use of a word notorious: The police caught one of the most notorious thieves last night. In this case, the selective component of the word thief blocks the use of the word celebrated.

The necessity of taking into consideration of the national-cultural selective subcomponent is determined by the inseparable connection of lexical meaning with the culture of the people and the specifics of their social life. It is hardly possible to explain the principle of combining such collocations as to make mistakes, to start a fire, to deliver lectures, heavy supper, to take measures, etc. These combinations are due to the national-cultural specifics of semantics.

\section{Types of information in the word semantics and collocability}

The modern approach to the study of the semantics of a word is based on the definition of its internal form as a complex formation, which is a structure of the lexical meaning of a word and consists of a number of components, called differently by different researchers, e.g. information types, sign parameters, aspects of meaning, macrocomponents, etc. The isolation of components reveals the real elements of the meaning that objectively exist in the word. They are presented within the meaning as a whole, but for the purpose of a deep analysis of the structure of lexical meaning, the whole is divided into components on the basis of various oppositions.

Within the framework of the communicative linguistics, meaning is considered as a set of mega-, macro- and microcomponents. Megacomponents - the largest components of meaning, represent the lexical (reflection of extra-linguistic reality) and structurallinguistic (reflected in the meaning of linguistic reality) meaning of the word. Megacomponents in turn consist of macrocomponents - denotative and connotative components, in the structural-linguistic - functional and grammatical. The macrocomponents of the meaning consist of microcomponents (semes), reflecting the differential signs of the word denotation and distinguishing the meanings of words [6].

In the semiotic aspect C. Morris [7] distinguishes semantics - the relationship between the sign and the subject, syntactics - the relationship between the sign and other signs, and pragmatics - the relationship between man and the subject, which can manifest as a subjective attitude to the subject and have emotional, expressive, stylistic, social information.

Within the framework of combinatorial semasiology, which studies the relationship between the semantics of a word and its collocability, it makes sense to distinguish denotative, connotative and syntagmatic macrocomponents of meaning, where the latter is designed to realize the collocability of words in speech. 
The syntagmatic macrocomponent seems significant, since, without entering into either the denotative or the connotative part of the meaning, it is responsible for the lexical and syntactic collocability of the word, and permits its use in certain constructions.

The syntagmatic macrocomponent includes semantic and non-semantic information that regulates the syntactic formation of a semantically flawless and normatively conditioned combination of a given word with another word.

The lexical meaning of the word contains not only a component that ensures its collocability with certain lexemes, but also a certain restrictive component, which, within certain limits, even prohibits their collocability. Such a case indicates the selection restrictions of the word, e.g. to drive a car, but not $*$ ship (to command), since these words have the so-called restrictive semes.

The syntagmatic macrocomponent contains the following microcomponents: 1) selective - allowing and limiting combinations of lexemes (to pour water because it is liquid); 2) prescriptive - causing a single focus of the phenomena of reality (conifer wood); 3 ) restrictive - double limiting of the collocability within a certain semantic class of words (an idea stroke me, but not a phenomenon* etc.).

The selective microcomponent is also complex and contains denotative-selective, which reflects ideas about objects and phenomena of the surrounding reality (listen to music, white wine, etc.); connotative-selective, which reflects the assessment, feelings, emotions and stylistic conditionality (an arrant scoundrel, to stare wide-eyed, etc.); national-cultural selective component that determines the collocability of words that reflect some cultural specificity of the language community (reception centre, amen corner, mystery tour, etc.).

The prescriptive microcomponent closely correlates with the narrow or mandatory collocability - the possibility of a word to be combined with one, two or three lexemes. This is a type of a very narrow range of collocability presented, for example, in such combinations of words as torrential rain. According to the theory of general phraseology, this collocation has an integral connotative meaning, so the content of it is reduced to one meaning.

In the framework of the contextological theory, there are examples of extreme narrowness of lexical collocability - "constant context" - a fixed connection of words in which variations of components are generally excluded or under certain conditions limited at most. Then the collocability of words is limited by their obligatory phraseological coherence with only one or two words. Words in stable collocations do not contain denotative meaning, because over time they have lost it, e.g., to harrow the soil.

\section{Actualization of a word in context}

The meaning of a word is formed under the influence of extra-linguistic conditions, while the meaning of a statement is the result of a purely linguistic factor - the context, where the word gets its actualization: low music, low water, low force, low cost, etc.

The phenomenon of actualization is inseparably connected with the collocability, since this is the adapting of virtual elements of the language to the requirements of this speech situation through actualizers, or linguistic means, by which these virtual elements of the language system relate to the reality of verbal communication. That is a word gets certain function, its real meaning in this particular context reveals [8].

The main role in the actualization of the meaning of the word is played by the context, which can be immediate (the words surrounding the given word) and broad, i.e. the entire text. Moreover, the use and understanding of a word is influenced by a communicative situation, or a combination of extralinguistic conditions. The meaning of the word in the context undergoes significant changes.

There are several functions of the context: it indicates the meaning of a polysemous word; it reveals the actual meaning, i.e. a specific set of semes that are actualized in a specific context. The meaning of the word includes all nuclear and peripheral semantic components. In speech, these components may not be fully implemented, but partly depending on the context and situation [9]. We should emphasize that the semes included in the actual meaning can be both nuclear and peripheral. Nuclear semes, which are the most vivid and reflected in the dictionary definition of the lexeme, are automatically contextualized.

In addition, there are contexts, in which the assemantic use of a word is possible, i.e. the use of the word without updating its meaning, or zero actual meaning: autonomous usage of the word, where no single component is actualized, because the word appears without its content, e.g. a pot is a three-letter word. Furthermore, there are purely expressive usages of words, which cannot be segmented into semes, e.g. "You're not Brad Pitt".

The context underlines peripheral semes through their explication and reduplication, building it in such a way that the peripheral semes manifest themselves through obligatory explicit duplication e.g. I am a hospitable person, I love to bake my specialty cake for guests. In this example, the collocability of words creates a context that underlines often peripheral semes through their explication and reduplication.

Context can add semes to the word to realize the communicative intention or modify them. To achieve the comic effect, the context can actualize several meanings at the same time.

The phrases that are formed as a result of filling in the syntactic positions equally reflect the specificity of the denotation of this particular word. The degree of typicality of the phrase is dependent on the status of semes that are actualized. It is important that the more specific semes are actualized in a word in some combination, the more characteristic this combination is.

The collocations with a given word, in which the semes are actualized, reflecting the specificity of its denotation, means its own collocability, while the totality of phrases with a given word, in which semes, 
reflecting not the most specific sides and properties of its denotation, is an improper collocability, i.e. the type of collocability characterizes not a separate word, but its belonging to a certain lexico-semantic group.

However, the actualization extends not only to the denotative component of the meaning of the word, but also to the potential emotionally-evaluative elements of the meaning by the possible enrichment of the content of a word through the interaction of two or more meanings: due to the conceptual or emotional associations.

\section{Culturally specific collocations}

The choice of words to send some idea to the listener or reader is important, but to decode the message without the knowledge of cultural differences is impossible. There is an example of a collocation fringe benefit, which means something that you get for working not in the form of money (a car, insurance, etc.). Knowledge of these two words separately cannot help a person with understanding of the whole.

The national diversity of linguistic cultures presupposes the manifestation of signs of ethnicity in the semantics of the language. The differences in people's ideas about the reality testify to various ways of conceptualizing and verbalization by different nations. The study of the national-cultural specifics of the semantics of words and their combinatorial-syntagmatic properties is due to the need to eliminate communicative barriers between representatives of different cultures. Therefore, it is necessary to point out the importance of considering the transfer of cultural-specific collocations, non-equivalent words and phrases, to another language.

Attention to the reflection of the relevant features for a certain linguistic group in the semantics of the word is quite explicable - national specifics is fixed and manifested in the language in the connotation of the word, collocation or sentence.

Special national-cultural environment becomes noticeable when confronted with the phenomena of another culture, so it is necessary to consider nonequivalent collocations on the material of several languages.

In the context of this study, we call non-equivalent those lexical units, whose relevant components are impossible or difficult to reproduce at the similar level of expression [10]. These lexical units suggest an indication of the fact that there is no equivalent in the input language, and also the reason for its absence, which makes them national-specific and allows to include in the concept of "non-equivalent vocabulary" units with national-cultural and national-linguistic features. The experience of a people is fixed in the language by means of word combinations, which are the syntactic basis for the formation of more complex unities, equivalent to a single word. The stabilization of a whole combination of words meets the needs of communication.

Ethnocultural collocations - phrases characteristic of a certain linguistic group, reflecting socially significant phenomena of reality (e.g. draft dodger - a person, who evades compulsory military service, legal separation - a legal process by which a married couple may formalize the separation while remaining legally married, cash bar - a bar at a party or event at which guests can buy drinks, happy hour - a period of time, when restaurants and bars offer discounts on alcoholic drinks, etc.) [11].

Ethnocultural collocations do not have units of a similar level within a pair of languages, which cannot be equivalent to different variants of reproduction of this unit by means of another language, i.e. they are characterized by the absence of a lexical unit with the same referential meaning or its incompleteness within a pair of languages. Hence, ethnocultural collocations is a special type of phrases with syntactic-semantic unity of combined words connected by a tradition of native speakers and reflecting significant elements for a particular society, which makes it difficult for non-native speakers to understand them. Ethnocultural collocations are unique formations that can be identified only within a pair of languages, in other words, in a comparative or contrastive aspect.

\section{Conclusion}

The lexical meaning as a complex unity contains components of different character, in particular syntagmatic subcomponent, carrying information on national peculiarities, taking part in the process of making collocations. Collocability of words performs several functions, such as demarcation of polysemous words, homonyms, it actualizes the semantic features of words, creates comic effect, etc.

Language fixes different outlook of people, their way of conceptualizing the reality. Signs of cultural specificity are shown in the semantics of the words, which enhances the creation of ethnocultural collocations - combinatorially conditioned collocations, reflecting significant realities for particular nation. Therefore, this type of collocation provokes interest and is necessary to be thoroughly analyzed.

\section{References}

1. D. Shmelyov, The Russian Language in a National School, Collocation of Words in the Russian Language, , 4, 72-73 (1961)

2. E. Arkhipova, M. Vlavatskaya, Advances in Intelligent Systems and Computing, Combinatorial linguodidactics as a new direction in the methodology of foreign language teaching, 907, 9197 (2019)

3. M. García-Page, Fraseología contrastiva con ejemplos tomados del alemán, español, francés e italiano, Universidad de Murcia, Colocaciones simples y complejas: diferencias estructurales, 145167 (2005)

4. W. Zuidema, B. Boer, Current Opinion in Behavioral Sciences, The evolution of combinatorial structure in language, 21, 138-144 (2018)

5. O. Anishcheva, Typology of Objective Lexical Collocability of the Verbs of Creation and Destruction, 20 (1981) 
6. I. Sternin, Problems of analyzing the structure of the meaning of the word, 155 (1979)

7. C. Morris, Foundations of the theory of signs. 13-71 (1938)

8. O. Akhmanova, Dictionary of linguistic terms, (Editorial URSS, Moscow, 2004)

9. I. Sternin, M. Solomatina, Semantic analysis of a word in context, 92 (2011)
10. A. Ivanov, Non-equivalent vocabulary, (University Press, St. Petersburg, 2006)

11. E. Arkhipova, Procedia - Social and Behavioral Sciences, The non-equivalent word-combinations selection for ideographic dictionary compiling, 387392 (2015) 